\title{
Determination of forces in the elements of the brake rigging of bogies of freight cars
}

\author{
Israil Elyazov ${ }^{1}$, Vasyl Ravlyuk ${ }^{2, *}$, Andriy Rybin ${ }^{2}$, and Vitalii Hrebeniuk ${ }^{2}$ \\ ${ }^{1}$ Azerbaijan Technical University, Department of Railway Transport Operation, 25 H. Javid Ave., Baku, Az 1073, Azerbaijan \\ ${ }^{2}$ Ukrainian State University of Railway Transport, Department of Cars, 7 Feuerbach Sq., Kharkov, 61050, Ukraine
}

\begin{abstract}
The article presents the results of studies the purpose of which was solving the problem of deceleration of abnormal wear of brake pads in freight cars of Ukrzaliznytsia JSC. In the studies, the design schemes of brake rigging during braking were considered theoretically. Particular attention was paid to the determination of force loads acting in the rods of the rigging and the contact area of the brake pads with the rolling surfaces of the wheels during braking. Design analysis was performed to determine rational solutions from the point of view of determined force load of the rigging elements of the bogies during braking, in particular, taking into account the action of harmful torque caused by the movement of the bogie on inequalities "track joints". Based on the conducted studies, it was decided to create a 2D generalized model diagram to determine reliable information on the operation of triangle brake rigging.
\end{abstract}

\section{Introduction}

Today, the main task of Ukrzaliznytsya JSC is increasing the volume of freight transportation, which requires increasing the weight and the speed of freight trains. This can only be achieved provided the brakes are failure-proof and that timely maintenance and repair of freight cars is performed. However, the maintenance conditions of the brake equipment of freight cars have deteriorated significantly in recent years. This is a major factor hampering the increase in the volume of freight transportations by the Ukrainian railways.

According to the inspection of the braking systems in the three-element bogies under operating conditions, it was found that the multiple pads in operation have excessive tilt when the brakes are released. Their upper ends rest against the rolling surface of the wheels which causes friction during the movement of the car (Fig. 1, a). Thus, two wear planes are formed on the working surface of the pad: the upper one, on which the pad wears obliquely symmetrically (Fig. 1, b); and the lower one which is used for braking.

In turn, it should be noted that such wedge dual pads lead to the occurrence of thermomechanical damage to the rolling surface of the wheels during braking (Fig. 1,c) which compromises the traffic safety and increases the repair costs.

In view of the above, in Ukrzaliznytsia JSC and in the leading universities related to railway transport the work is being done to improve the reliability of the brake systems of freight trains.

Until now, 2D model diagrams of operation of brake rigging (BR) of various designs have been commonly considered in practice; they were used by the authors as a basis to propose a generalized mathematical design model for which theoretical studies with corresponding calculations have been performed.
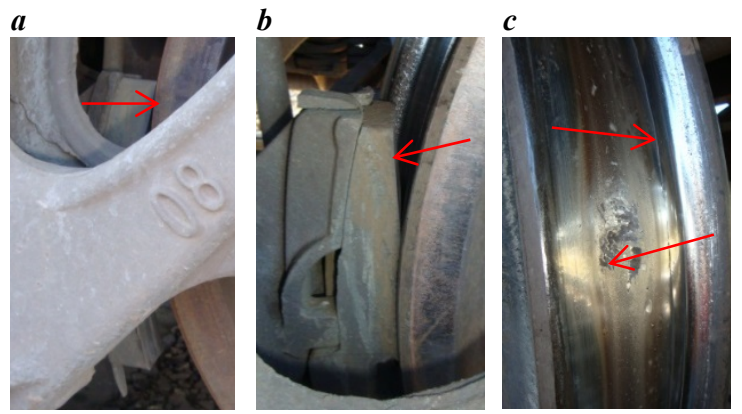

Fig. 1. Defect on wheels caused by abnormally worn brake pads: a - interaction of the upper end with the rolling surface of the wheel during movement of the car; $b$ - braking with the wedge-worn pad; $\mathrm{c}$ - thermomechanical defects on the rolling surface of the wheel due to the increased braking time.

\section{Analysis of recent research and publications}

The study of scientific, technical and promotional sources of information on the operability of BR of modern freight cars proves that none of the developed rigging designs of bogies [1, 2, 3] can solve the problem because the developers proposed to create various auxiliary devices for counteracting forces which make the pads tilt until they touch the wheels in the bogies only on the basis of kinetostatic analysis of the BR mechanism [4].

\footnotetext{
* Corresponding author: ravvg@ukr.net
} 
In [5] it was determined that the BR configuration currently used in two-axle freight cars, even with the brake pads of maximum thickness, cannot ensure their wear to the minimum permissible value in operation without additional manual adjustment. However, the authors of the article do not cover the issue of the wedge dual wear of the brake pads. In [6], a method of estimation of brake pad presses was developed, which includes structural analysis of the BR mechanism, determination of position of elements based on the coordinate method, and force analysis in which the BR is considered as a static flat mechanism. Studies were conducted at nominal brake pad sizes and different wheel diameters, but issues related to wedge dual friction wear and its effect on the braking force were not considered. In [7] the coordination of the dimension chains of BR of a freight car is substantiated in order to determine the nature and magnitude of wear of the brake pads of freight cars. A method for calculating the size chains of BRs is proposed, which allows determining the limit deviations of the component elements for the brake pads, which wear evenly.

In [8], the dynamic forces effecting the load-bearing structure of freight car bodies and related to the safe movement during operation in international traffic were analyzed, but the issue of change of the three-element bogie braking system was not considered.

In [9] the stresses occurring in the brake pad were analyzed, the thermal analysis using SolidWorks software was performed and an alternative solution for improving the brake pad material and increasing their life was suggested.

The authors in [10] refer to various friction brake systems used in mechanical braking. It is noted that friction brake systems in which brake pads are used have adverse effect on the rolling surface of the wheels, as high temperatures occur in the "pad-wheel" friction zone, so disc brakes are preferred

Using conventional methods of research, a dimensional analogue model with finite elements of the brake block on wheels was developed in [11], which was combined through a contact interface to control of the heat produced during braking, as well as heat distribution on the surface of the wheel block through thermal contact supports. The effect of temperature in wheels and brake blocks during braking was analyzed in experimental studies on a brake test bench in $[12,13]$.

The analysis of the literature suggests that the problems of operation of the brake systems of the threeelement bogies in which the wedge dual wear of brake pads occurs have not been given sufficient attention.

\section{Purpose and tasks of the study}

The purpose of this study is creating a $2 \mathrm{D}$ generalized brake rigging model which will be used to find a rational solution to neutralize the effect of harmful torque in the triangle brake systems of bogies of freight cars.

To achieve the stated purpose, the following tasks must be solved:

- considering 2D design model diagrams during braking of three-element bogies of freight cars for different design solutions of the brake rigging;

- developing a generalized model of modern brake rigging of freight cars which will allow interpreting its operation in the form of non-inertial and inertial 2D kinematic schemes;

- performing calculations of the force factors effecting the rigging elements during braking of the bogie for various cases, to be used as the basis for finding a rational solution for reducing the harmful effect of torque, as well as to take into account the operational conditions of the freight car when the bogies roll over the inequality "rail joint";

- proposing actions to neutralize the effect of harmful torque which upsets a balance of the triangle of the brake rigging of the three-element bogie of the freight car.

\section{Results and discussion}

To reduce the possible effect of the harmful torque caused by the dynamics of the movement of freight cars, let us consider the BR triangles of three-element bogies and analyze ways to improve them. Fig. 2 shows a model bogie BR of a freight car. Investigating the structure of this mechanism makes it clear that it has redundant connections, so determining kinematics and performing its dynamic analysis immediately; finding inertial force factors affecting the BR from its parts is also impossible. As a result, kinetostatic analysis of this complex mechanism requires either imposing certain constraints, or considering its dynamics using a second-order Lagrange equation.

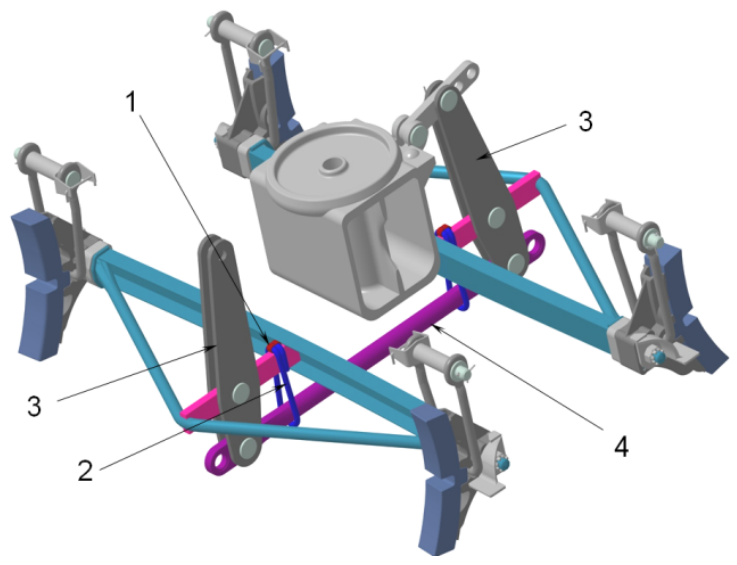

Fig. 2. General view of the BR model of the four-axle bogie of the freight car: 1 - locking device; 2 - bracer for even wear of pads; 3 - vertical lever; 4 - bridle.

If the first option is chosen, it is advisable to consider, for design reasons, that in operation of "symmetrical" BR, the force presses of the brake pads on the wheels are numerically identical. Then the spatial designs of freight car bogies with BR can be reduced to 2D braking diagrams, and in the future this will be considered for only one wheel pair.

Let us conditionally divide the BR of the bogie into two parts, which are attributed to the first (right) and the second (left) wheel pair (Fig. 3). The first part (the first wheel pair) has triangle No. 1, with a leading vertical 
lever through which along with other BR parts braking force $T_{0}(t)$ is transferred from the pneumatic brake system and through lever 1 activates triangle No. 1, which with its brake shoes with pads 4 presses the wheels of the first wheel pair and brakes it. At the same time, the effect of forces on the first lever, which balance reaction of $T_{1}$ from the pressure forces of the pads of triangle No. 1 on the wheels; force $\mathrm{N}_{1}$ in the pendulum suspensions of triangle No. 1, as well as internal force $\delta T$, which cannot be found immediately by the conventional method of forces due to the excessive degree of freedom of BR [14].

The second part (the second wheel pair) has its triangle No. 2, the vertical (driven) lever 2, to which an undetermined internal force $\delta T$ is transmitted through the spacer of both levers in the opposite direction.

To understand the principle of operation of the triangular BR, it is sufficient to focus on a static analysis of the actions of the above power factors, without taking into account weight of its parts. It is clear that the geometry ratio of the parameters of the symmetric BR can be chosen so that pressing of the brake pads on the wheels of each of the two wheel pairs in their contact areas were numerically identical, i.e. reaction $T_{1}=T_{2}$.

In the diagram (Fig. 3), some external forces and moments are shown in the red arrows for better perception of the scheme, while internal reactions in the spacer of vertical levers are shown in yellow.

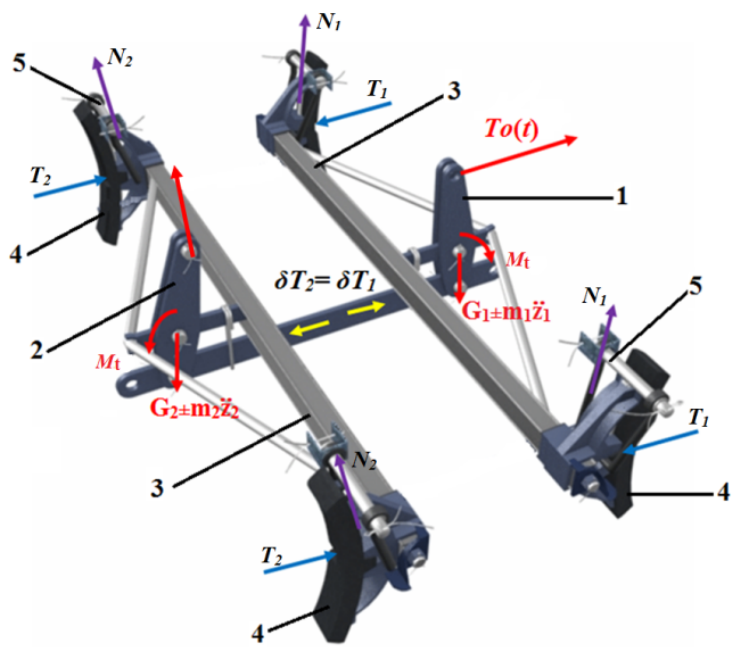

Fig. 3. General view of the BR model when force $T_{0}(t)$ is transmitted during braking of the freight car: 1,2 - vertical lever; 3 - triangle; 4 - brake pad; 5 - pendulum suspension.

Classical mechanics problems which involve equilibrium equations of the chosen model (statics, kinematics with dynamics elements and dynamics in the form of Lagrange equations of the second kind) can be solved using different methods. It depends on the formulation of the problem, which in our case is reduced to a static bar system. However, these can be solved using either the force, or displacement, or hybrid method. The force method will be applied for further designing.

Earlier studies [4] analyzed in detail the problem associated with the design of BR bogies and the excessive wear of their brake pads. In this article, we investigated the case of reducing the weight of the BR triangular structure to zero (non-inertial system). The calculations established a decrease of the base pressure values of the pads on the rolling surface of the wheels, which corresponds to the expected decrease in the intensity of their wear (Fig. 4, a). However, this result can only be achieved theoretically. In addition, braking safety conditions are violated in this case, which is unacceptable.
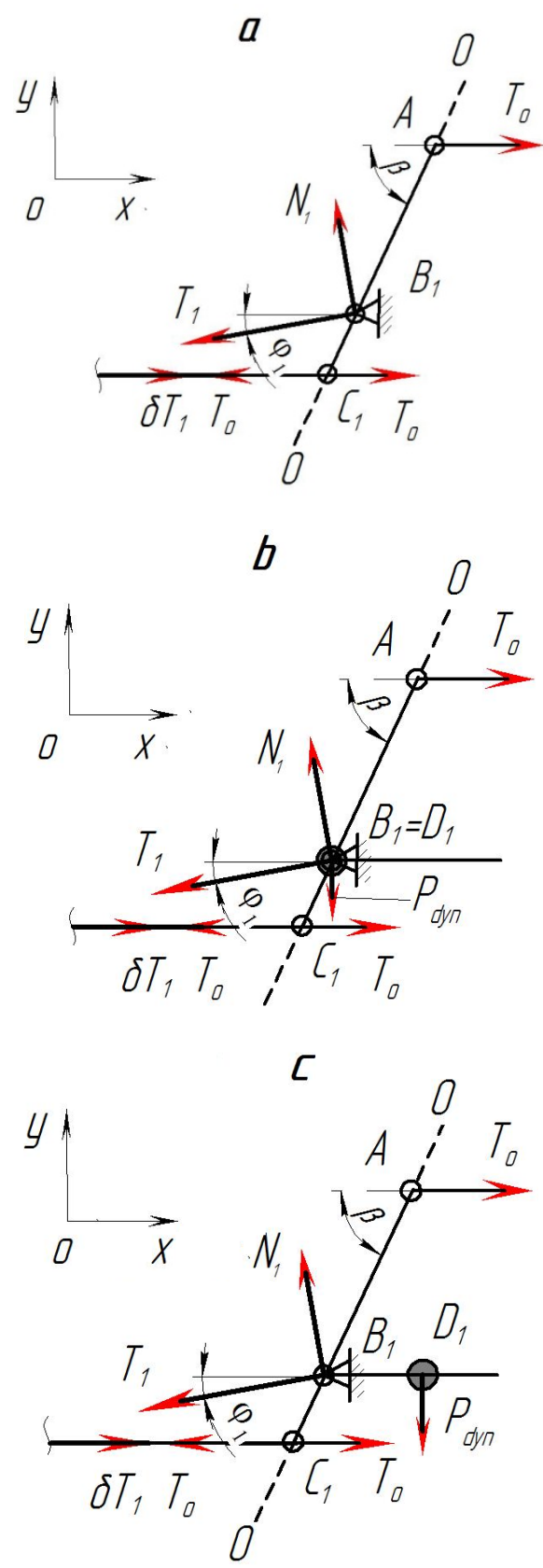

Fig. 4. Calculation model diagrams of the braked first (right) wheel pair when it rolls over the inequality "rail joint" (phase 2).

The operation of BR was theoretically considered, where, ideally, its centre of mass and the connection knot of the triangle spacer with the leading lever (Fig. 4, b) are initially experimentally reduced to a single 
straight line (suspension axis of the triangle). Although in this case the desired result regarding the calculations of force actions in all links of the triangular BR seems to be achieved, it is very difficult to achieve in practice.

In this sense, a real model diagram (Fig. 4, c) was considered separately without using auxiliary devices and without any significant changes in the typical triangle design.

Here, effect of harmful torque $M_{t}$ was reduced and the problem of deceleration of the wear rate of the brake pad was partially solved. Upgraded BR triangles became more efficient than typical brakes of wheel pairs, mainly due to the action of the braking force $P_{o}(t)$, normally occurring during braking [15]. However, without an ingenious theoretical analysis of the operation of triangle BRs, the results of search for design solutions are discrepant as to the reduction of the harmful effects of $M_{t}$, there are only not substantiated (approximate) solutions. Indeed, for triangle No. 1, which is suspended to the bogie by two pendulum suspensions for brake shoes with first wheel pair pads, a rational solution in this regard is when the centre of mass $C_{l}$ (Fig. 4, c) is on axis $O-O$.

In the authors' opinion, the "perfect" BR design among the considered ones is the model (Fig. 4, a), where there is a non-inertial triangle, i.e., there are no dangerous stress factors.

The model (Fig. 4, b) can be considered to be close to the reality. Here, the combined center of gravity of the triangle is on its carrier beam. Then the influence of dangerous force factors is minimal (tending to zero). However, such a solution requires the installation of auxiliary balances behind the carrier beam on the other side of the triangle, which is next to impossible.

The other model is realistic and associated with the maximum possible displacement of the opening to connect the vertical lever to the brake strut as close as possible to its beam (Fig. 4, c and Fig. 5). This design solution was patented.

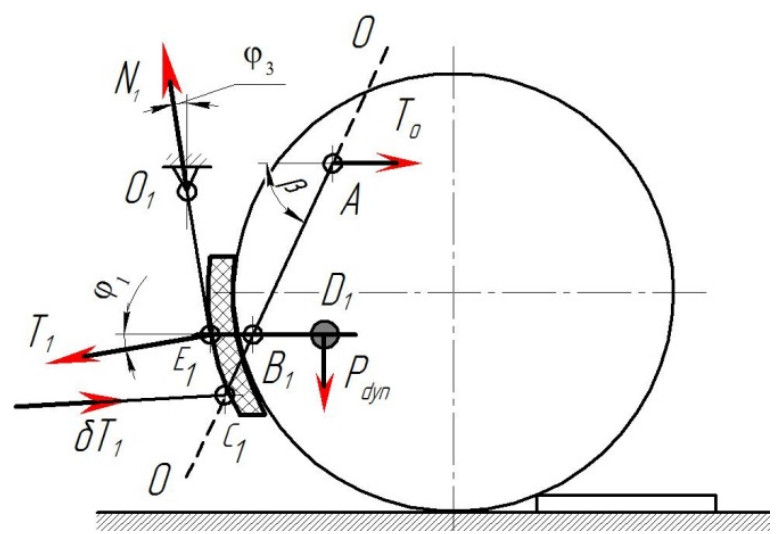

Fig. 5. Combined 2D model diagram for braking the right wheel pair rolling over the inequality "rail joint".

\subsection{Theoretical calculation method of analysis of operation of BR}

Concerning general movement and braking of a freight train, let us consider the phases of movement of a separate wheel pair of a bogie: no braking - phase 0 ; braking with movement - phase 1 , or without any movement (full service braking) - phase 2 .

Let us consider phase 0 . In the released state of the brake system, the proposed design changes to the triangle include retaining the brake pads without tilting to the rolling surfaces of the wheels to prevent harmful upper wedge dual wear of the pads.

However, since the statically balanced triangle is held in such a "free" state in its suspension joints by friction forces only at the hinge joints, it cannot be ruled out that vibration of the undercarriage of the moving car on the track inequalities may from time to time make the triangle with the shoes and pads tilt in one direction or the other. In this case, the upper parts of the edges of pads in the released state due to the above vibration touch the rolling surface of the wheels.

It should be noted that, in general, the combined centre of mass of the triangle with its attached BR parts cannot be stationary at any particular point, at least because during the "free" movement, unlike braking, the centres of mass of each element of BR change their locations. Therefore, in the comparative analysis of the action of force factors on BR elements in various design schemes, it is appropriate to consider braking phase 3 with the intermediate location of the total centre of mass $C_{1}$ of the triangle with its parts [16].

From the point of view of kinetostatic analysis of BR operation, where the force factors act on the components of the brake system of the bogie in the classic situation of the bogie hitting isolated irregularity $\eta$ or on other irregularities of the track, the kinematic diagrams of BR will have different forms and correspond to more than one phases of use of the rigging.

Approximate solutions to this problem for different 2D model diagrams can be obtained using the generalized scheme (Fig. 5), where it is shown that during the modernization of the triangle with the restoration of its spacer in the depot, a positive result was obtained, which was verified (Fig. 4, b).

Let us draw a generalized model diagram of operation of the triangle BR during braking (phase 2) from which all the above follow.

Let us consider phase 2. Supposing a BR bogie brakes in Phase 1 or Phase 2, and the combined centre of gravity of triangle No. 1 of the right wheel pair with the attached elements (probably point $C_{1}$ ) first crosses the space above the "rail joint" [14].

Then there is a formalized calculation scheme of the braked first (right) wheel pair when it roll over the inequality "rail joint" taking into account the effect of gravitational force (Fig. 5).

According to the condition of the use of a typical brake in a freight car, the effect of force factors on the $\mathrm{BR}$ is reproduced as follows. Under the action of external force $T_{0}(t)$, which changes together with the change in pressure in the brake pneumatic cylinder, an initial impulse to the first braking phase appears, where this force increases its value to $T_{0}$ and causes strain of forces $N_{l}$ in the suspension rods of triangle No. 1 .

At the same time, force $T_{0}$ is divided to virtual force $\delta T$, which together with it will press the wheels of the 
first and second wheel pairs through the pads. Virtual force $\delta T$ in general can move the spacer connecting levers 1 and 2 (Fig. 3) either forward (right) or back (left) $[14,16]$.

In the end of the braking phase, when the pads of triangles No. 1 and No. 2 already rest against the wheels of the first and second wheel pairs, and the car is either slowing down or stops at all.

Let us consider the static equilibrium equation for $\mathrm{BR}$ (general case) and get separate solutions for the right part of the bogie with BR (Fig 5).

These mathematical models (1) and (2) are valid for the case of unilateral pressing of the pads on the wheels, the standard values of the angles of the pads were also taken into account (Table 1).

$$
\sum X=0 ; T_{0}+\delta T-N_{1} \sin \varphi_{1}-T_{1} \cos \varphi_{1}=0 ;
$$

$$
\sum Y=0 ; \quad N_{1} \cos \varphi_{1}-T_{1} \sin \varphi_{1}-P_{d y n}=0 ;
$$

$$
\begin{aligned}
& \sum M_{B_{I}}=0 ; T_{0} \cdot l_{B_{I} C_{I}} \cdot \sin \beta-P_{d y n} l_{B_{I} D_{I}}- \\
& \quad-N_{I} \cdot l_{B_{I} E_{I}} \cdot \cos \varphi_{I}-T_{I} \cdot l_{B_{I} E_{I}} \cdot \sin \varphi_{I}+ \\
& \quad+\delta T \cdot l_{B_{I} C_{I}} \cdot \sin \beta-M_{0}=0,
\end{aligned}
$$

where $M_{0}$ is compensating moment which occurs when the pad presses on the wheel and is $M_{0}=T_{0} \cdot l_{A C_{1}} \cdot \sin \beta$; $P_{d y n}$ is inertial force which occurs in the triangle when the wheel hits the obstacle and moves the triangle with the pads towards the wheel (on the basis of the previous studies, it is assumed $\left.P_{d y n}=1 \mathrm{kN}\right) ; l_{B_{1} C_{1}}, l_{\mathrm{B}_{1} \mathrm{D}_{1}}$, are geometrical parameters of BR elements (Table 1).

Table 1. Input parameters for calculations

\begin{tabular}{|c|c|c|}
\hline Designation & Value, $\mathbf{m m}$ & Value, $\mathbf{m}$ \\
\hline$l_{B_{1} C_{1}}$ & 160 & 0.16 \\
\hline$l_{B_{1} D_{1}}$ & 150 & 0.15 \\
\hline$l_{B_{1} O_{1}}$ & 50 & 0.05 \\
\hline$l_{C_{1} A}$ & 560 & 0.56 \\
\hline$\varphi_{1}$ & $10^{0}$ & $\begin{array}{c}\cos \varphi_{1}=0.984, \\
\sin \varphi_{1}=0.1736\end{array}$ \\
\hline$\beta$ & $64^{0} 43^{\prime}=64.72^{0}$ & $\begin{array}{c}\cos \beta=0.427, \\
\sin \beta=0.904\end{array}$ \\
\hline
\end{tabular}

Equations (1) - (3) are easy to transform in the system of equations of the general form (4):

$$
\left\{\begin{array}{c}
T_{0}+\delta T-N_{1} \sin \varphi_{1}-T_{1} \cos \varphi_{1}=0 \\
N_{1} \cos \varphi_{1}-T_{I} \sin \varphi_{1}-P_{d y n}=0 \\
T_{0} \cdot l_{B_{I} C_{I}} \cdot \sin \beta-P_{d y n} l_{B_{I} D_{I}}-N_{I} \cdot l_{B_{I} E_{I}} \cdot \cos \varphi_{I}- \\
-T_{1} \cdot l_{B_{I} O_{I}} \cdot \sin \varphi_{1}+\delta T \cdot l_{B_{I} C_{I}} \cdot \sin \beta-M_{0}=0
\end{array} .\right.
$$

After vector of unknown forces $\Delta=\left(N_{1}, T_{1}, \delta T\right)$ is introduced, the matrix of the equation system will have the form $G$, in which the right side of the matrix has the form of the column vector $R$ :

$$
\begin{gathered}
G=\left[\begin{array}{ccc}
-\sin \varphi_{I} & -\cos \varphi_{1} & 1 \\
-\cos \varphi_{I} & -\sin \varphi_{1} & 0 \\
-l_{B_{I} E_{I}} \cdot \cos \varphi_{1} & -l_{B_{I} O_{I}} \cdot \sin \varphi_{I} & l_{B_{I} C_{I}} \cdot \sin \beta
\end{array}\right], \\
R=\left[\begin{array}{c}
-T_{0} \\
P_{d y n} \\
-T_{0} \cdot l_{B_{I} C_{I}} \cdot \sin \beta+P_{d y n} \cdot l_{B_{I} D_{I}}+T_{0} \cdot l_{A C_{I}} \cdot \sin \beta
\end{array}\right] .
\end{gathered}
$$

To solve the system of equations by the Kramer method, the matrix determinant $G=\Delta$ should be found. Unless it is zero, the system of linear algebraic equations has a unique solution.

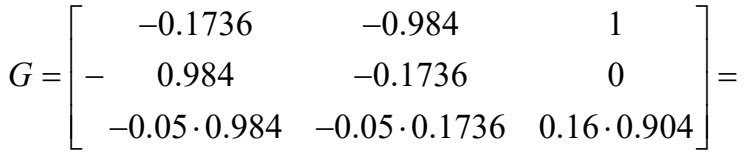

$$
\begin{aligned}
& =\left[\begin{array}{ccc}
-0.1736 & -0.984 & 1 \\
-0.984 & -0.1736 & 0 \\
-0492 & -0,00868 & 0.14464
\end{array}\right] \\
& R=\left[\begin{array}{c}
-13.4 \\
1 \\
-13.4 \cdot 0.16 \cdot 0.904+1 \cdot 0.15+13.4 \cdot 0.56 \cdot 0.904
\end{array}\right]= \\
& =\left[\begin{array}{c}
-13.4 \\
1 \\
4,99544
\end{array}\right]
\end{aligned}
$$

Forces to be found will be calculated for each moment of time using the formulas:

$$
N_{1}=\frac{\Delta_{1}}{\Delta}, \quad T_{1}=\frac{\Delta_{2}}{\Delta}, \quad \delta T=\frac{\Delta_{3}}{\Delta},
$$

where $\Delta_{1}, \Delta_{2}, \Delta_{3}$ are determinants of matrices in which according to the index of column $(1,2,3)$ of the matrix (5), the systems are replaced by a column of the matrix (6).

$$
\begin{aligned}
& N_{1}=\frac{1.3546814976}{0.14444075577344}, \\
& T_{1}=\frac{6.84676864}{0.14444075577344}, \\
& \delta T=\frac{5.0373317761024}{0.14444075577344} .
\end{aligned}
$$


Practice or experiment is known to be the criterion for the adequacy of any design model. In our case, the operational testing of the upgraded BR of freight cars has shown that improved performance ensures the specified wear of the brake pads and increases their service life.

\subsection{Analysis of calculation schemes for designing BR}

The plan of positions of BR during braking in phase 2 for the bogie of the 18-100 freight car is considered. All necessary initial data for obtaining solutions are given in Table 1. Force factors which exist in the components of bogie BR were found using the Mathcad software (Table 2).

Table 2. Comparison of identified forces acting in the BR elements

\begin{tabular}{|l|c|c|c|c|}
\hline Calculated scheme & $\boldsymbol{T}_{\mathbf{1}}, \mathbf{k N}$ & $\boldsymbol{T}_{\mathbf{2}}, \mathbf{k N}$ & $\boldsymbol{N}_{\mathbf{1}}, \mathbf{k N}$ & $\boldsymbol{N}_{\mathbf{2}}, \mathbf{k N}$ \\
\hline Typical & 46.0 & 46.0 & - & - \\
\hline Scheme a) & 45.53 & 45.53 & 8.03 & 8.03 \\
\hline Scheme b) & 40.97 & 40.97 & 7.13 & 7.13 \\
\hline Scheme c) & 47.4 & 47.4 & 9.8 & 9.8 \\
\hline United & 47.413 & 47.413 & 9.381 & 9.381 \\
\hline
\end{tabular}

The obtained calculations prove that the design changes of BR, which reflect the considered model diagrams, have significant differences with respect to the determined force loads of the elements of the triangular BRs.

It was also found that a shift of the technological opening of the triangle spacer can be considered the simplest rational solution, which can be used primarily in the "optimization' of the operating conditions of the braking system of the bogie, and accordingly, the train. This is achieved in a technologically accessible and economically feasible way in the facilities of the car repair enterprise and does not require significant capital investments.

The second step in the BR design improvement is definitely reduction of the total mass of the triangle with its elements and the dynamic balancing of its design which is associated with search for the "optimal" location of its centre of mass.

\section{Conclusions}

On the basis of the conducted studies, the following conclusions can be drawn:

1. 2D model diagrams were calculated for various design solutions of brake rigging, which enabled to identify the cause and solve the problem of deceleration of abnormal wear of the brake pads of freight cars.

2. According to the results of theoretical studies, a generalized model diagram of operation of triangle BRs was created and various schematic solutions were considered with their scientific substantiation.

3. Comparative design analysis was performed to determine rational solutions in terms of determined force load of elements of the brake rigging of the bogies during braking, taking into account the harmful torque. 4. The first step in terms of BR modernization was scientifically substantiated, which was achieved in a technologically accessible and economically feasible way at the facilities of the car repair enterprise and does not require any significant capital investments. On the basis of the results of the studies it was decided to create a $2 \mathrm{D}$ generalized model diagram to determine reliable information on the operation of triangular rigging transmissions of bogies of freight cars.

\section{References}

1. G. Vrtanoski, T. Smileski, Dynamic testing of innovative railway brake system for freight wagons. ANNALS of Faculty Engineering Hunedoara International Journal of Engineering 17, 83-89 (2019)

2. E.P. Blohin, K.T. Alpysbaev, V.Y. Panasenko, ZK1 bogies of gondola cars built in China. Wagon park 9(66), 12-14 (2012)

3. I.V. Turutin, E.A. Rudakova, Design of the 18-9889 and 18-9890 bogies for innovative four- and six-axle freight cars. Transport of the Russian Federation 3(46), 10-12 (2013)

4. V.G. Ravlyuk, Simplified kinetostatic analysis of brake brake rigging of freight cars. Proceedings of the State University of Infrastructure and Technology 32, 55-70 (2018)

5. S.V. Tuluzin, D.V. Gorskij, Performance assessment of the brake rigging of the bogie of the freight car at various stages of wear of pads and wheels. Bulletin VNIIZHT 2, 38-44 (2015)

6. V.A. Karpychev, G.B. Nikitin, P.A. Andreev, Issue of evaluation and control of braking efforts of pads on the wheels depending on the positions of the levers when adjusting the rigging of the 18-100 bogie. Bulletin VNIIZHT 5, 43-48 (2013)

7. A.V. Smolyaninov, P.V. Smolyaninov, Dimensional calculations of the brake rigging of a freight car as a method of substantiating techniques to improve the quality of repair. Izvestia Transsib 2(10), 27-36 (2012)

8. O. Fomin, A. Lovska, V. Radkevych, A. Horban, I. Skliarenko, O. Gurenkova, The dynamic loading analysis of containers placed on a flat wagon during shunting collisions. ARPN Journal of Engineering and Applied Sciences 14(21), 3747-3752 (2019)

9. O.C. Ambikaprasad, A.R. Abhijeet, Failure Analysis of Brake Shoe in Indian Railway Wagon. IPASJ International Journal of Mechanical Engineering 3, 37-41 (2015)

10. R.C. Sharma, M. Dhingra, R.K. Pathak, Braking systems in railway vehicles. International Journal of Engineering Research \& Technology 4, 206-211 (2015)

11. T. Vernersson, Temperatures at railway tread braking. Part 1: Modeling, Proceedings of the 
Institution of Mechanical Engineers. Part F. Journal of Rail and Rapid Transit 221, 167-182 (2007)

12. K.P. Vineesh, M.R.K. Vakkalagadda, A.K. Tripathi, A. Mishra, V. Racherla, Non-uniformity in braking in coaching and freight stock in Indian Railways and associated causes. Engineering Failure Analysis 59, 493-508 (2016)

13. M.R.K. Vakkalagadda, D.K. Srivastava, A. Mishra, V. Racherla, Performance analyses of brake blocks used by Indian Railways. Original Research Article 328-329, 64-76 (2015)

14. V.P. Shpachuk, V.O. Pushnja, O.I. Rubanenko, A.O. Gharbuz, Compendium of lectures "Theoretical Mechanics. Dynamics" (KhNUMG named after O.M. Beketov, Kharkiv, 2016)

15. V. Ravlyuk, Modernization of the elements of the brake rigging of bogies of freight cars. Nauka ta progres transportu. Bulletin of Dnipropetrovsk National University of Railway Transport named after Academician V. Lazaryan 5(83), 108-121 (2019)

16. A.N. Koptovets, L.N. Shirin, E.M. Shlyakhov, A.V. Denishchenko, V.V. Zil, V.V. Yavorskaya, Simulation of friction processes in the operation of pad wheel brake of underground locomotives (State Higher. educational institution "National Mining University", Dnipro, 2017) 